\title{
An Improved Adaptive Bilateral Filter to Remove Gaussian Noise from Color Images
}

\author{
${ }^{1}$ Manjeet Kaur ${ }^{2}$, Shailender Gupta and ${ }^{3}$ Bharat Bhushan \\ Electronics and Communication Engineering Department, \\ YMCA University of Science and Technology, Faridabad, India \\ 1'mkgrewal31@gmail.com, ${ }^{2}$ shailender81@gmail.com, ${ }^{3}$ bhrts@yahoo.com
}

\begin{abstract}
Introduction of noise in image causes variation in brightness or colour information. Various noises such as Gaussian, Impulse, Speckle, Periodic andPoisson noise deteriorates the picture quality. Gaussian noise is the most popular one that incurs during acquisition process caused by poor illumination and/or high temperature. This paper proposes an improved modified adaptive Bilateral Filter to remove Gaussian noise from colour images. This technique is implemented in MATLAB-9 and various performance metrics taken into consideration are: Peak Signal to Noise Ratio (PSNR), Mean Square Error (MSE), Mean Absolute Error (MAE), and Normalized Colour Difference (NCD). The result shows that the proposed technique gives the best results than all other techniques in terms of all comparison parameters.
\end{abstract}

Keywords: Bilateral filter, Gaussian noise, NCD value, noise removal, range filter, sharpness enhancement

\section{Introduction}

Any degradation in the image due to external disturbance can be called as noise[15]. There are various reasons for introduction of noise in the image. The main types of noises are discussed below:

1. Salt and Pepper Noise: This noise is introduced due to sharp and sudden disturbance in the image signal. It appears as white or black (or both) pixels scattered randomly over the image. It is also called impulse noise or binary noise.

2. Gaussian Noise: Gaussian noise is an idealized form of white noise. White noise is a random signal with flat power spectral density. Gaussian noise is caused due to random fluctuations in the signal. It is also called additive noise as it can be modeled by random values added to an image.

3. Poisson Noise: Poisson noise is also called photo shot noise, as it is caused by random variations of photons, which cause photons to enter one sensor than the other.

4. Speckle Noise: This noise can be modeled by random values multiplied by pixel values, hence it is called multiplicative noise. It causes major problem in some radar applications.

5. Periodic Noise: This noiseis introduced due to periodic disturbance in the image signal. It is created by cyclic processes such as machines, engines etc. It is commonly caused by interference between electronic components.

These noises affect the image quality differently. The effect of the introduction of above mentioned noises on images is shown in Table 1. 
International Journal of Signal Processing, Image Processing and Pattern Recognition Vol.8, No.3 (2015)

Table 1. Effect of Addition of Noises on Picture Quality

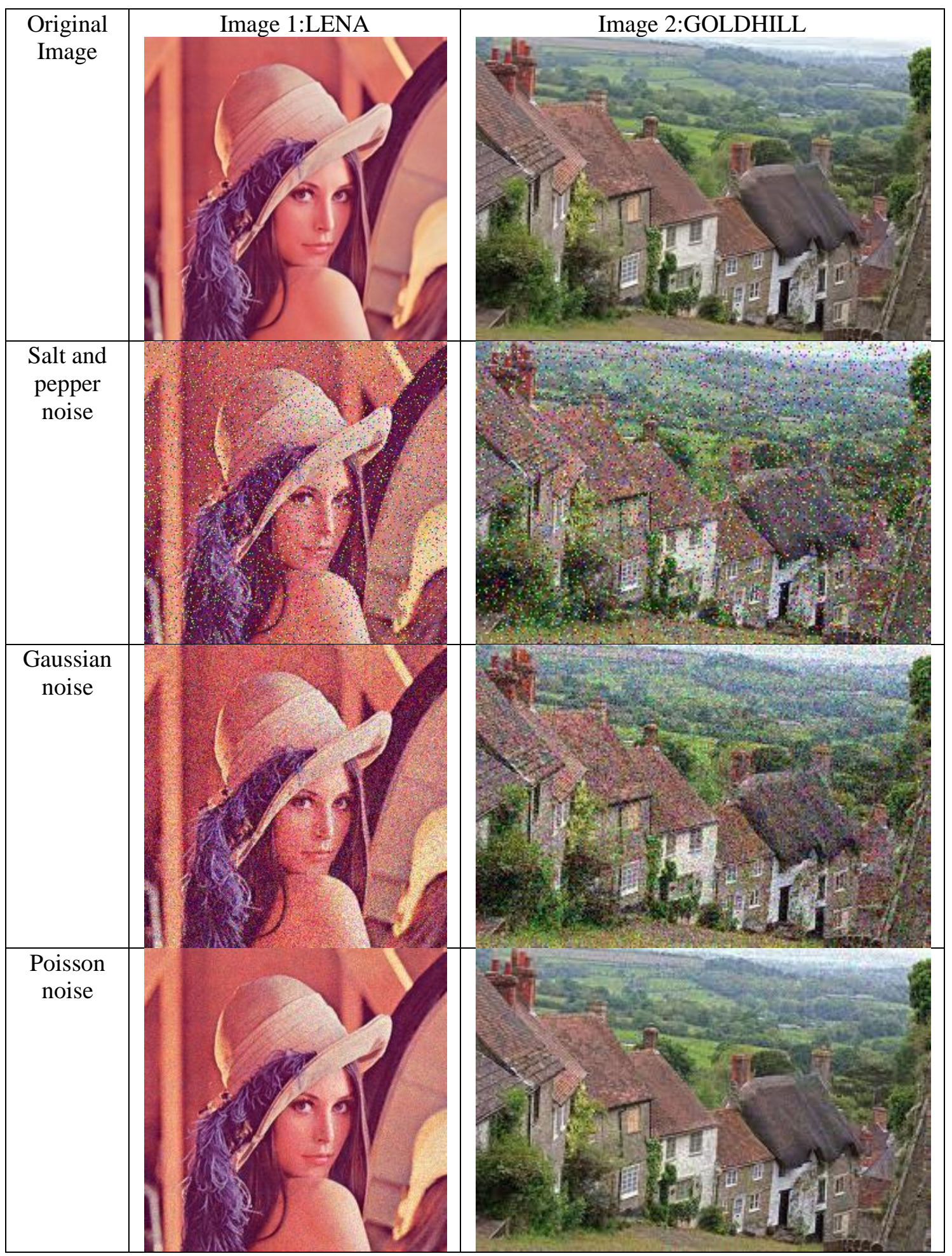




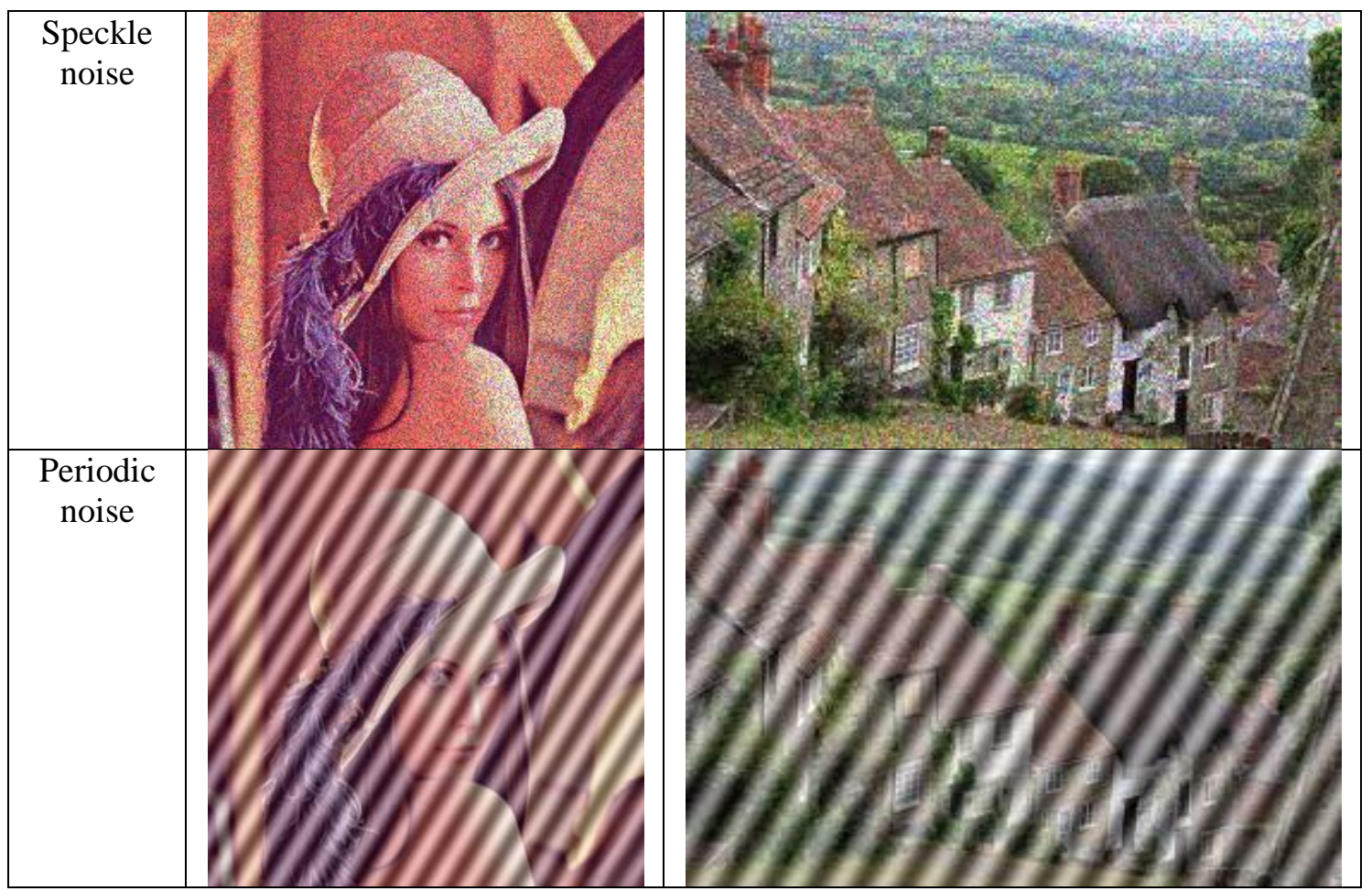

This paper deals with the removal of Gaussian noise, the most common form of noise which degrades the image quality. To remove Gaussian noise various filtering techniques are proposed in literature. In this paper we implemented Bilateral Filter, Modified Bilateral Filter (MBF), Joint Bilateral Filter (JBF), Fuzzy Bilateral Filter (FBF), and Switching Bilateral Filter (SBF) for the comparison with the proposed Modified Adaptive Bilateral Filter (MABF). The rest of the paper is organised as follows. In section II, literature survey and problem identification is given. Section III presents the proposed technique and the simulation setup parameters used in implementation of filtering techniques. Section IV presents the performance metrics taken into consideration for comparison. In Section V , results of comparison of performance and visual quality is given. Finally the conclusion is given in Section VI.

\section{Literature Survey and Problem Formulation}

1. Bilateral Filter: The bilateral filter proposed by Tomasi and Manduchi[1] is the combination of range and domain filtering. It adopts a low pass Gaussian filter for both range and domain filtering. As presented in [12] if $\left[\mathrm{m}_{0}, \mathrm{n}_{0}\right]$ is the center pixel of window defined, $\sigma_{\mathrm{d}}$ and $\sigma_{\mathrm{r}}$ are the standard deviations of the domain and range Gaussian filters, respectively, and

$$
r_{m_{0}, n_{0}}=\sum_{m=m_{0}-N} \sum_{\mathrm{n}=\mathrm{n}_{0}-\mathrm{N}}^{\mathrm{m}_{0}+\mathrm{N}} \mathrm{e}^{\left(-\frac{\left(m-m_{0}\right)^{2}+\left(n-n_{0}\right)^{2}}{2 \sigma_{d}^{2}}\right)} \times \mathrm{e}^{\left(-\frac{\left(g[m, n]-g\left[m_{0}, n_{0}\right]\right)^{2}}{2 \sigma_{r}^{2}}\right)}
$$

is the kernel weight function or normalisation factor. The domain low pass Gaussian filter compares the other pixels with the centre pixel in window and assigns higher weights to 
pixels that are especially close to centre pixel. Similarly, range low pass Gaussian filter assigns higher weights to pixels which are similar to centre pixel in grey value.

The results of bilateral filter show that it reduces the noise significantly but does not sharpen the edges. Hence in order to enhance the sharpness we need to modify the bilateral filter and therefore an attempt is made in this paper to improve the results of bilateral filter. In addition to the standard bilateral filter we also implemented other bilateral filtering techniques proposed in literature to remove Gaussian noise and then compared the results in terms of PSNR, MSE, MAE and NCD values.

2. Fuzzy Bilateral Filter: In FBF [5-6] a particular class of fuzzy metrics is used to represent the spatial and photometric relations between the colour pixels adapting the classical bilateral filtering [1]. In the first step, two fuzzy metrics are used; the first one to measure the photometric fuzzy distance between colour vectors and the second one to measure the spatial fuzzy distance between the pixels under comparison. In this filter the weight vector is calculated as follow:

$$
w(i, j, t)=\prod_{s=1}^{3} \frac{\min \left\{F_{i}^{s}, F_{j}^{s}\right\}+K}{\max \left\{F_{i}^{s}, F_{j}^{s}\right\}+K} \times \frac{t}{t+\|i-j\|_{2}}
$$

By using this weight vector the FBF output is calculated as

$$
\widetilde{F}_{l}=\frac{\sum_{j \in W} w(i, j, t) F_{j}}{\sum_{j \in W} w(i, j, t)}
$$

Where $F_{i}$ and $F_{j}$ are the pixels under the window at $\mathrm{i}^{\text {th }}$ and $\mathrm{j}^{\text {th }}$ locations and $\mathrm{t}$ is the parameter to adjust the output. The value of $\mathrm{K}$ is 1024 for RGB image. It is a very fast and simple method for removing Gaussian noise from images but it blurs the image.

3. Joint Bilateral Filter: The joint bilateral filter $[4,14]$ is basically an extension of bilateral filter in which two correlated images, one taken with flash and other without flash are used for filtering process. The non-flash image is sometimes referred as the ambient image. In joint bilateral filter the flash image is used to compute range filter kernel rather than the ambient image. The illumination from a flash may change appearance of the scene due to brightness, it also increases the signal-to-noise ratio and provides much better estimate of true highfrequency information than the ambient image, hence the flash image is called an estimator. In [4] after detecting flash shadows, the basic bilateral filter is applied within those regions. The results of joint bilateral filter show that it preserves the detail while reducing noise.

4. Modified Bilateral Filter: In modified bilateral filter [12], each pixel from the filtering window $\mathrm{W}_{\mathrm{x}}$ is assigned a minimum connection cost of a path that joins them with the central pixel $x$. The connection cost is a function of absolute differences of pixel intensities. For finding the minimum cost paths we apply the Dijkstra algorithm. The cost of a path is the sum of connection costs of adjacent pixels forming a path. The connection cost is used to calculate a weight assigned to each pixel from $\mathrm{W}_{\mathrm{x}}$ and the filter output is the weighted average of the surrounding pixels of $\mathrm{x}$. The weights are defined as:

$$
w(x, y)=\exp \left(-\frac{C(x, y)^{2}}{h^{2}}\right)
$$

Where $h$ is a tuning parameter. The range of $h$ is [150,250]. The default value of $h$ is taken as 200. $\mathrm{C}(\mathrm{x}, \mathrm{y})$ is a cost function of the minimum path connecting $\mathrm{x}$ and $\mathrm{y}$. For color images the connection costs are calculated using the Euclidean distance in RGB color space between neighboring pixels. 
5. Switching Bilateral Filter: The SBF removes noise without adding another weighting function $[7,10]$. The operation is performed in two stages: detection followed by filtering. For detection the sorted quadrant median vector (SQMV) scheme is used, which includes important features such as edge or texture information. During detection process a status is assigned to each pixel such that, whether it is noise-free or noisy. If pixel is found noisy it is replaced by a proper median value obtained by edge detector in the range filter function according to the following weight function as in bilateral filter:

$$
w(x, y)=w_{d}(x, y) w_{s r}(x, y)
$$

Except that here $w_{s r}(x, y)$ is calculated as:

$$
W_{S R}(x, y)=e^{-\frac{1}{2}\left(\frac{I_{x}-F y}{\sigma_{d}}\right)^{2}}
$$

Where $I_{x}$ is $F_{x}$ if it is found Gaussian noise otherwise it is replaced by a reference median which is calculated in detection process. This technique performs well for removal of gaussian noise from images.

\section{The Proposed Technique}

Bilateral filter [1] is a smoothing filter, it does not restore the sharpness of a degraded image. To enhance the results of bilateral filter, we proposed a modified adaptive bilateral filter. The adaptive bilateral filter [8] is the general form of bilateral filter. The important modifications done in bilateral filter to increase the picture quality of de-noised image are:

1. An offset $\zeta$ is introduced to the range filter.

2. Both $\zeta$ and $\sigma_{\mathrm{r}}$ i.e. the width of the range filter are made locally adaptive.

3. The CIE-Lab color space is used.

If $\left[\mathrm{m}_{0}, \mathrm{n}_{0}\right]$ is the center pixel of window defined, $\sigma_{\mathrm{d}}$ and $\sigma_{\mathrm{r}}$ are the standard deviations of the domain and range Gaussian filters, respectively, then the kernel weight function or normalisation factor used in the proposed technique can be defined as:

$$
r_{m_{0}, n_{0}}=\sum_{m=m_{0}-N} \sum_{\mathrm{n}=\mathrm{n}_{0}-\mathrm{N}}^{\mathrm{m}_{0}+\mathrm{N}} \mathrm{e}^{\left(-\frac{\left(m-m_{0}\right)^{2}+\left(n-n_{0}\right)^{2}}{2 \sigma_{d}^{2}}\right)} \times \mathrm{e}^{\left(-\frac{\|\left(g[m, n]-g\left[m_{0}, n_{0}\right]-\zeta\left[m_{0}, n_{0}\right] \|^{2}\right.}{2 \sigma_{r}^{2}\left[m_{0}, n_{0}\right]}\right)}
$$

By making $\zeta$ and $\sigma_{\mathrm{r}}$ adaptive and jointly optimizing both the bilateral filter is transformed into much more powerful and versatile filter. To find the pair of parameters which minimizes the MSE, an exhaustive search is performed in the parameter space $\phi=\left\{\left(\zeta, \sigma_{\mathrm{r}}\right): \zeta \in \phi_{\zeta} \& \sigma_{\mathrm{r}} \epsilon\right.$ $\left.\phi_{\sigma_{r}}\right\}$,

Where $\phi_{\zeta}=[-60,60]$ and $\phi_{\sigma_{r}}=[5,45]$. The range and step size of the parameters are chosen empirically such that they can yield adequate sharpening and smoothing for all types of images.

The feature we have chosen for pixel classification is the strength of edges measured by a Laplacian of Gaussian (LoG) operator which can be defined as:

$\operatorname{LoG}[\mathrm{m}, \mathrm{n}]=\left\{\begin{array}{cc}-\frac{1}{\pi \sigma_{\text {LoG }}^{4}}\left(1-\frac{m^{2}+n^{2}}{2 \sigma_{\text {LoG }}^{2}}\right) \exp \left(-\frac{m^{2}+n^{2}}{2 \sigma_{\text {LoG }}^{2}}\right)-C,|m|,|n| \leq N \\ 0, & \text { else }\end{array}\right.$ 
and $\mathrm{C}=\frac{1}{(2 \mathrm{~N}+1)^{2}} \sum_{m=m_{0}-N}^{m_{0}+N} \sum_{n=n_{0}-N}^{n_{0}+N}-\frac{1}{\pi \sigma_{\mathrm{LoG}}^{4}} \times\left(1-\frac{\mathrm{m}^{2}+\mathrm{n}^{2}}{2 \sigma_{\mathrm{LoG}}^{2}}\right) \exp \left(-\frac{\mathrm{m}^{2}+\mathrm{n}^{2}}{2 \sigma_{\mathrm{LoG}}^{2}}\right)$

Where $\sigma_{\mathrm{LoG}}=1.5$ and $\mathrm{N}=4$.

The LoG operator [15] has following characteristic properties:

1. It is a high pass filter.

2. It computes the second derivative of input image.

3. Response: Near edges: magnitude of its response is high

In smooth regions: magnitude of its response is low

On the center of an edge: magnitude of its response is 0 .

The pixel class index is computed according to

$$
L[m, n]=\left\{\begin{array}{lr}
{\left[g_{L o G}[m, n]\right],} & \left|g_{L o G}[m, n]\right| \leq L_{\max } \\
L_{\max }, & g_{L o G}[m, n]>L_{\max } \\
-L_{\max }, & g_{L o G}[m, n]<-L_{\max }
\end{array}\right.
$$

where $g_{\text {LoG }}[m, n]=\operatorname{LoG}[m, n] * * g[m, n], L_{\max }=60$ and $[x\rfloor$ denotes rounding $\mathrm{x}$ to the nearest integer. $\zeta$ is approximately equal to the LoG class. The desired amount of offset is proportional to the magnitude of the LoG response. In additional, the sign of $\zeta$ is the same as that of the LoG class. The flow chart of bilateral filtering technique and the proposed filtering technique is given in Figure 1 and Figure 2 respectively.

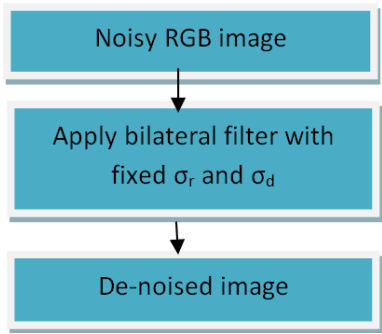

\section{Figure 1. Flow Chart of Bilateral Filtering Technique}

In basic bilateral filter the width of range filter i.e. $\sigma_{\mathrm{r}}$ is fixed. To make the bilateral filter more powerful and versatile we introduced offset $\zeta$, and both $\zeta$ and $\sigma_{\mathrm{r}}$ are made locally adaptive as shown in Figure 2 below.

FLOW CHART OF PROPOSED FILTERING TECHNIQUE: 


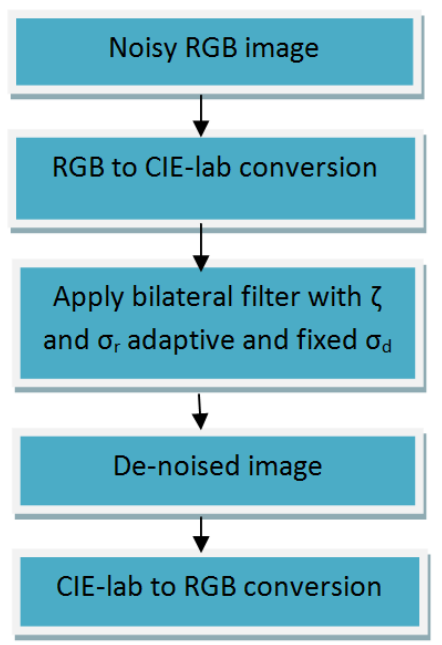

Figure 2. Flow Chart of Improved Adaptive BF

MATLAB is used as simulator to implement various filtering techniques. Various setup parameters used in simulation which are common to all techniques are as shown in Table 2.

Table 2. Setup Parameters

\begin{tabular}{|l|l|}
\hline Image size(pixels) & $\begin{array}{l}\text { LENA: 256*256*3 (color) } \\
\text { GOLDHILL: 275*183*3 (color) }\end{array}$ \\
\hline Image type & Jpg \\
\hline Gaussian Noise & Standard deviation (sigma) $=5,10,15,20,25,30$ \\
\hline Simulation Tool & MATLAB R2009a \\
\hline Processor & Intel(R) Core(TM) i3-3110M CPU $2.40 \mathrm{GHz}$ \\
\hline RAM & $2.00 \mathrm{~GB}$ \\
\hline
\end{tabular}

In the next section various performance metrics used in the simulation are defined.

\section{Performance Metrics for Comparison}

Peak Signal to Noise Ratio (PSNR): It is the measure of quality of the image by comparing denoised image with original image. It is an expression used to depict the ratio of maximum possible power of image (signal) and the power of the corrupting noise that affects the quality of its representation.

Mean Square Error (MSE): It is the cumulative squared error between the final de-noised image and the original image. This enables us to compare mathematically as to which method provides better results.

Mean Absolute Error (MAE): It is the absolute error between the original image and the denoised image. It represents the average value of introduced deviation per pixel with respect to original image. 
Normalized color difference (NCD): It is used to measure the degradation in color quality in color images since it approaches the human perception.

Perceptual Quality: Picture quality is a characteristic of an image that measures the perceived image degradation (typically, compared to an ideal or perfect image). Instead of denoised image should possess high PSNR and Low MSE, MAE; the de-noised image should be smooth, clean and clear also. De-noised image should be so fine for human observer as if it seems natural image. It should not have color blurriness or any odd looking structure.

\section{Results}

In this section the simulation results of filtering techniques as discussed above, are compared. Figures 3-6 show the results of PSNR, MSE, MAE, and NCD respectively.

\section{Results of PSNR:}

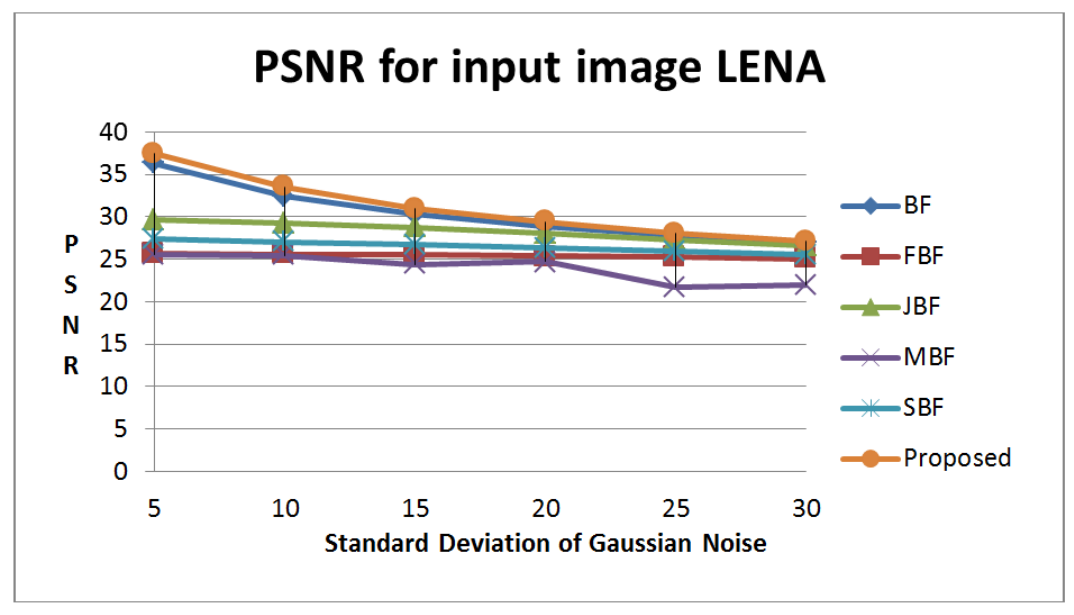

Figure 3(a). Comparison of PSNR Results for Input Image LENA

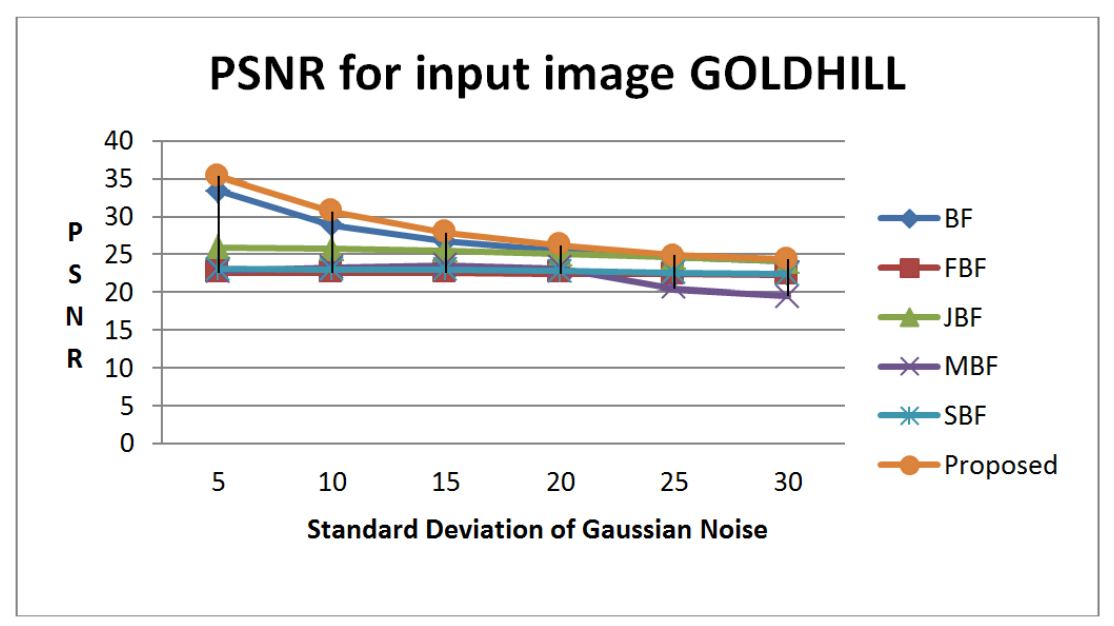

Figure 3(b). Comparison of PSNR Results for Input Image GOLDHILL

Figure 3. Comparison in Terms of PSNR 
In Figure 3 the results of PSNR for all variants of BF and proposed technique are shown for standard deviation from 5 to 30. From the results following inferences can be made:

- The proposed filtering technique gives the highest PSNR value when the image is corrupted with Gaussian noise.

- The MBF gives the lowest value of PSNR for large values of standard deviation whereas FBF gives lowest PSNR for small values of standard deviation of Gaussian noise.

Results of MSE:

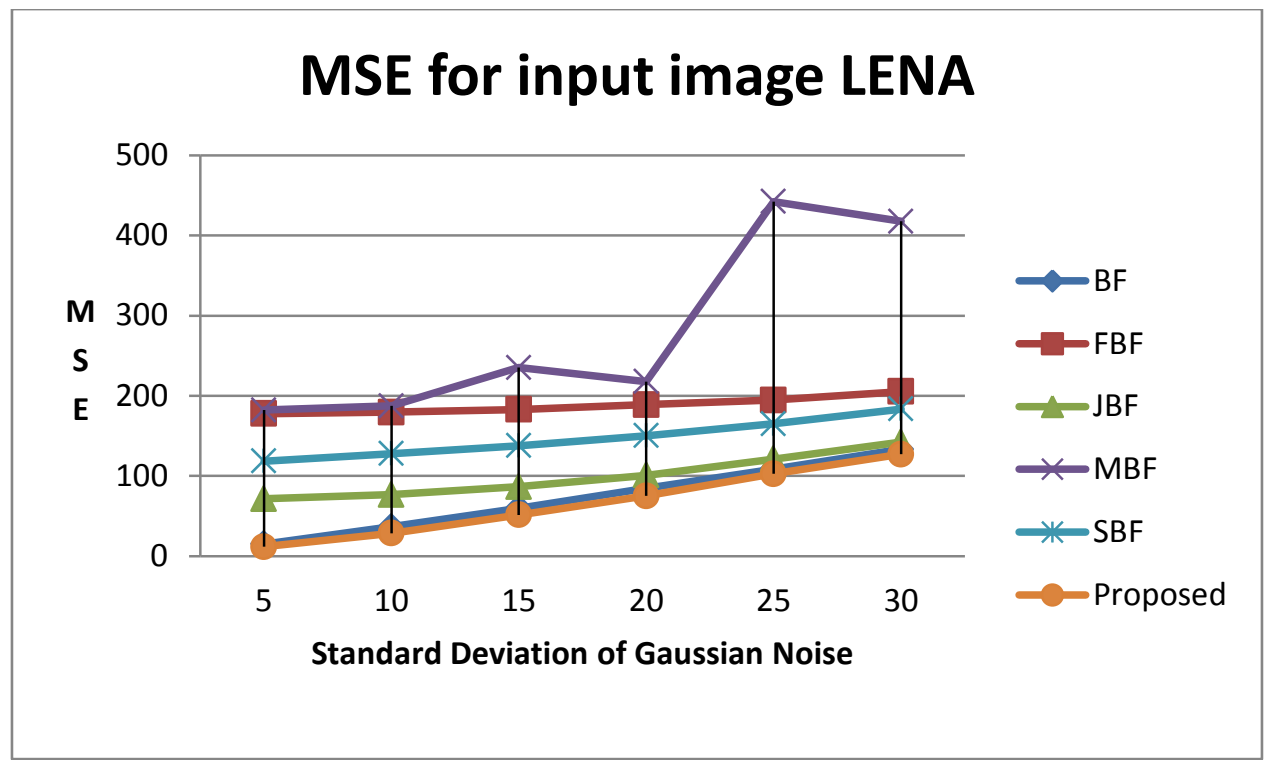

Figure 4(a). Comparison of MSE Results for Input Image LENA

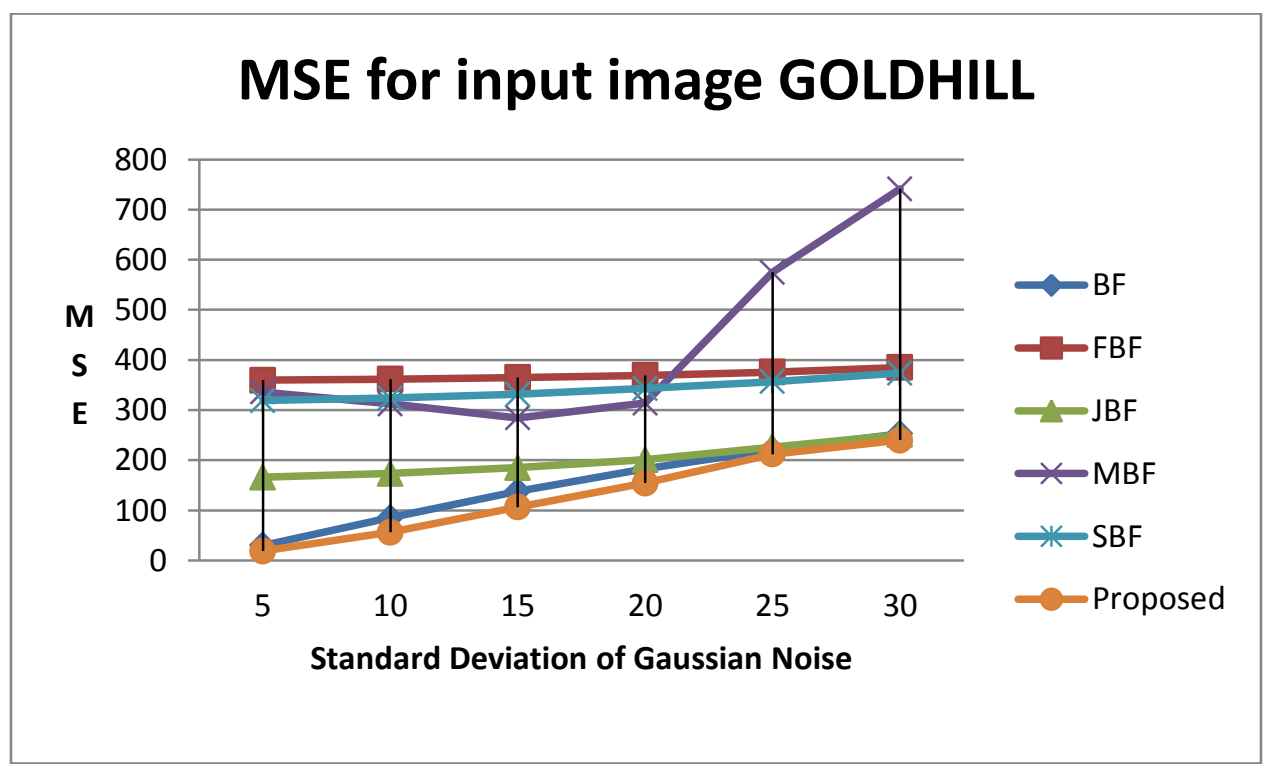

Figure 4(b). Comparison of MSE Results for Input Image GOLDHILL

Figure 4. Comparison in Terms of MSE 
In Figure 4 the results of MSE for all variants of BF and proposed technique are shown for standard deviation from 5 to 30. From the results following inferences can be made:

- The proposed filtering technique gives the lowest MSE value when the image is corrupted with Gaussian noise.

- The MBF gives the highest value of MSE for large values of standard deviation whereas FBF gives large MSE for small values of standard deviation of Gaussian noise.

\section{Results of MAE:}

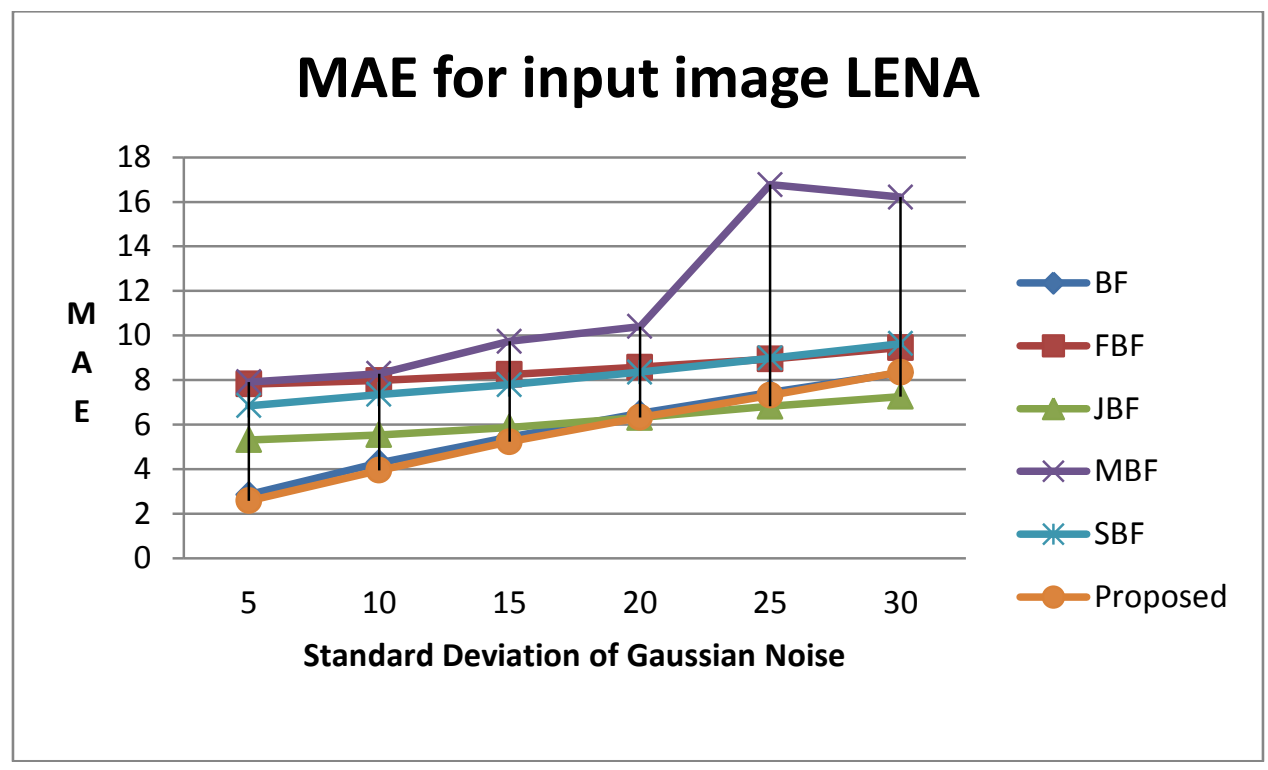

Figure 5(a). Comparison of MAE Results for Input Image LENA

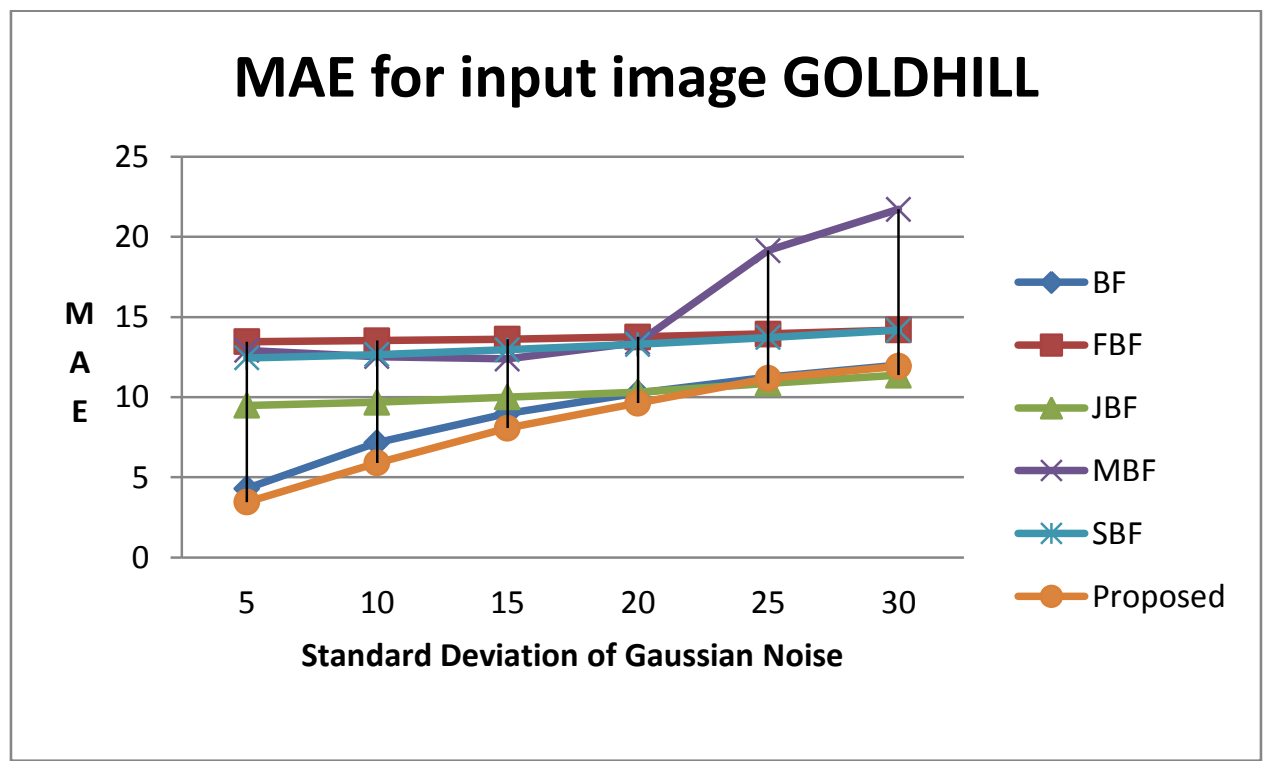

Figure 5(b). Comparison of MAE Results for Input Image GOLDHILL

Figure 5. Comparison in Terms of MAE 
In Figure 5 the results of MAE for all variants of $\mathrm{BF}$ and proposed technique are shown for standard deviation from 5 to 30. From the results following inferences can be made:

- The proposed filtering technique gives the lowest MAE value when the image is corrupted with Gaussian noise.

- The MBF gives the highest value of MAE for large values of standard deviation while FBF gives large MAE for small values of standard deviation of Gaussian noise.

Results of NCD:

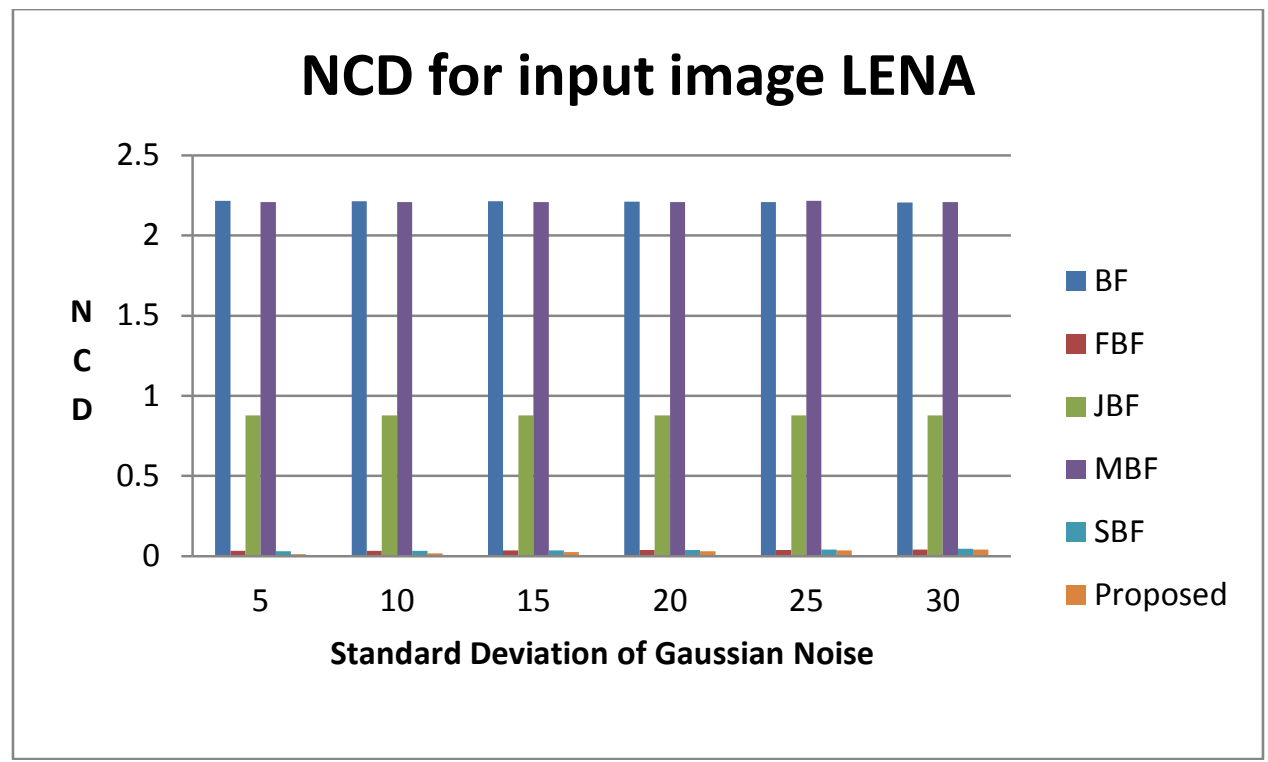

Figure 6(a). Comparison of NCD Results for Input Image LENA

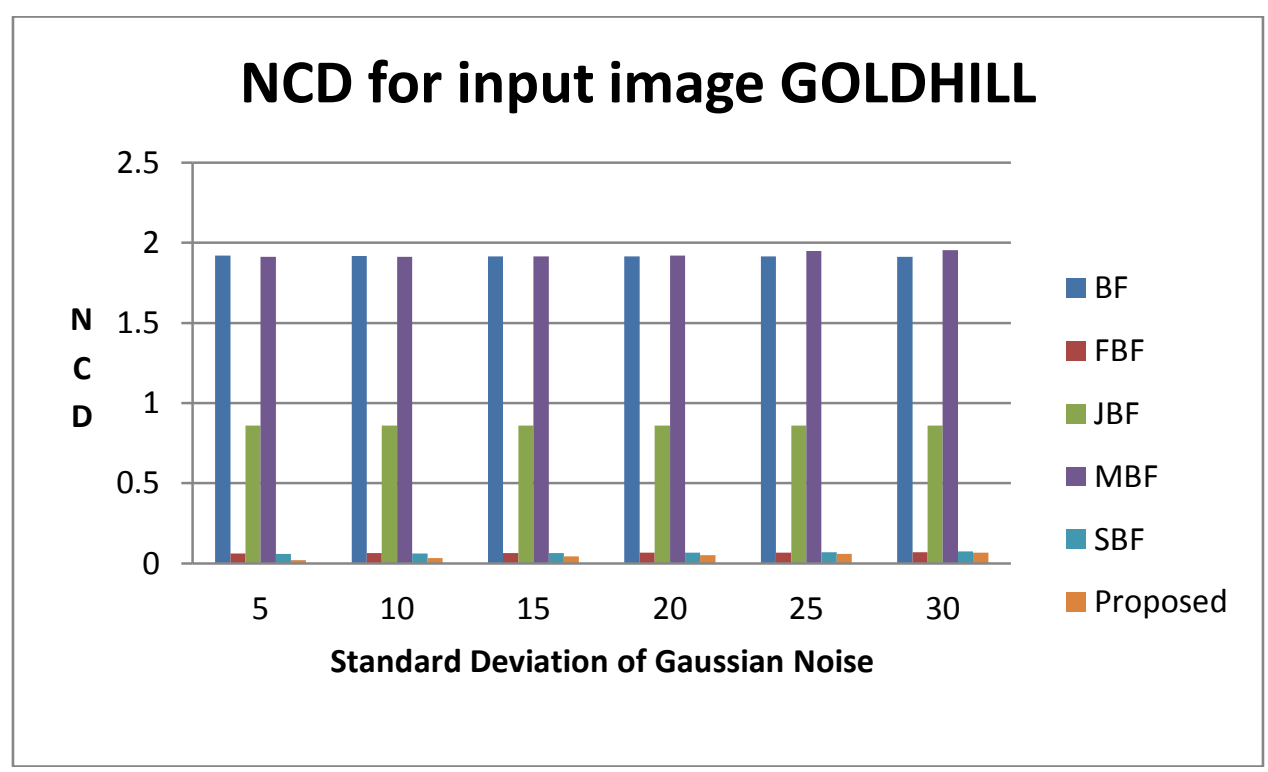

Figure 6(b). Comparison of NCD Results for Input Image GOLDHILL

Figure 6. Comparison in Terms of NCD 
In Figure 6 the results of NCD for all variants of BF and proposed technique are shown for standard deviation from 5 to 30. From the results following inferences can be made:

- The proposed filtering technique gives the lowest NCD value when the image is corrupted with Gaussian noise.

- The MBF gives the highest value of NCD for large values of standard deviation whereas BF gives large NCD for small values of standard deviation.

Also the overall results of all filtering techniques are given in Table 2 and Table 3 for $\sigma=5$ to 30 for LENA and GOLDHILL images respectively.

Table 2. PSNR, MSE, MAE and NCD for Gaussian Noise Removing Techniques (LENA)

\begin{tabular}{|c|c|c|c|c|c|c|c|}
\hline $\begin{array}{c}\text { Standard } \\
\text { Deviatio } \\
n\end{array}$ & $\begin{array}{c}\text { Paramete } \\
\text { r }\end{array}$ & $\mathrm{BF}$ & $\mathrm{FBF}$ & $\mathrm{JBF}$ & $\mathrm{MBF}$ & SBF & $\begin{array}{c}\text { PROPOSE } \\
\text { D }\end{array}$ \\
\hline \multirow{4}{*}{5} & PSNR & 36.3371 & 25.6333 & 29.5887 & 25.5266 & 27.394 & 37.4039 \\
\hline & MSE & 15.1138 & 177.7274 & 71.4837 & 182.1883 & 118.4899 & 11.8221 \\
\hline & MAE & 2.85 & 7.8168 & 5.3101 & 7.9141 & 6.852 & 2.5807 \\
\hline & NCD & 2.2163 & 0.0331 & 0.8783 & 2.2081 & 0.0308 & 0.0122 \\
\hline \multirow{4}{*}{10} & PSNR & 32.4866 & 25.5913 & 29.2849 & 25.4056 & 27.0632 & 33.5518 \\
\hline & MSE & 36.6796 & 179.4527 & 76.6646 & 187.2946 & 127.8665 & 28.701 \\
\hline & MAE & 4.2851 & 7.982 & 5.5431 & 8.2913 & 7.3571 & 3.944 \\
\hline & NCD & 2.2142 & 0.0341 & 0.8783 & 2.2076 & 0.0336 & 0.0188 \\
\hline \multirow{4}{*}{15} & PSNR & 30.3594 & 25.5059 & 28.7534 & 24.417 & 26.7406 & 31.0234 \\
\hline & MSE & 59.8607 & 183.0155 & 86.6442 & 235.1668 & 137.7281 & 51.3734 \\
\hline & MAE & 5.4402 & 8.2502 & 5.8789 & 9.7373 & 7.793 & 5.2301 \\
\hline & NCD & 2.2123 & 0.0355 & 0.8783 & 2.2072 & 0.0357 & 0.0248 \\
\hline \multirow{4}{*}{20} & PSNR & 28.8499 & 25.3701 & 28.0905 & 24.749 & 26.3646 & 29.3733 \\
\hline & MSE & 84.7404 & 188.8287 & 100.9319 & 217.8617 & 150.1831 & 75.1183 \\
\hline & MAE & 6.4993 & 8.5744 & 6.3173 & 10.407 & 8.3551 & 6.3212 \\
\hline & NCD & 2.2101 & 0.0374 & 0.8781 & 2.2082 & 0.0387 & 0.0303 \\
\hline 25 & PSNR & 27.7673 & 25.2373 & 27.2983 & 21.6747 & 25.954 & 28.0171 \\
\hline
\end{tabular}




\begin{tabular}{|c|c|c|c|c|c|c|c|}
\hline & MSE & 108.7306 & 194.6952 & 121.13 & 442.1911 & 165.0751 & 102.6538 \\
\cline { 2 - 8 } & MAE & 7.4394 & 8.9456 & 6.8179 & 16.7701 & 8.9783 & 7.3148 \\
\cline { 2 - 8 } & NCD & 2.2088 & 0.0394 & 0.8779 & 2.2157 & 0.042 & 0.0347 \\
\hline \multirow{3}{*}{30} & PSNR & 26.8812 & 25.0059 & 26.6069 & 21.9231 & 25.4966 & 27.0893 \\
\cline { 2 - 8 } & MSE & 133.3388 & 205.3491 & 142.033 & 417.609 & 183.4098 & 127.1014 \\
\cline { 2 - 8 } & MAE & 8.3239 & 9.459 & 7.2617 & 16.2088 & 9.6404 & 8.3574 \\
\cline { 2 - 8 } & NCD & 2.2052 & 0.0421 & 0.8779 & 2.2083 & 0.0455 & 0.0409 \\
\hline
\end{tabular}

Table 3. PSNR, MSE, MAE and NCD for Gaussian Noise Removing Techniques (GOLDHILL)

\begin{tabular}{|c|c|c|c|c|c|c|c|}
\hline $\begin{array}{c}\text { Standard } \\
\text { Deviatio } \\
\mathrm{n}\end{array}$ & $\begin{array}{c}\text { Paramete } \\
\mathrm{r}\end{array}$ & $\mathrm{BF}$ & $\mathrm{FBF}$ & $\mathrm{JBF}$ & $\mathrm{MBF}$ & SBF & $\begin{array}{c}\text { PROPOSE } \\
\text { D }\end{array}$ \\
\hline \multirow{4}{*}{5} & PSNR & 33.3898 & 22.5698 & 25.9263 & 22.8689 & 23.0955 & 35.35 \\
\hline & MSE & 29.7923 & 359.8319 & 166.1322 & 335.8877 & 318.8059 & 18.9705 \\
\hline & MAE & 4.302 & 13.4631 & 9.4861 & 12.9103 & 12.4479 & 3.4527 \\
\hline & NCD & 1.9206 & 0.0629 & 0.8605 & 1.9117 & 0.0605 & 0.0191 \\
\hline \multirow{4}{*}{10} & PSNR & 28.8174 & 22.5458 & 25.7403 & 23.1861 & 23.028 & 30.6419 \\
\hline & MSE & 85.376 & 361.8298 & 173.4 & 312.2267 & 323.8058 & 56.09 \\
\hline & MAE & 7.1589 & 13.5272 & 9.6806 & 12.5304 & 12.6609 & 5.9018 \\
\hline & NCD & 1.9168 & 0.0636 & 0.8605 & 1.9116 & 0.0626 & 0.0323 \\
\hline \multirow{4}{*}{15} & PSNR & 26.7264 & 22.5073 & 25.4567 & 23.5884 & 22.9254 & 27.8502 \\
\hline & MSE & 138.1791 & 365.0474 & 185.1032 & 284.6039 & 331.5452 & 106.6756 \\
\hline & MAE & 8.9785 & 13.6126 & 10.0021 & 12.3838 & 12.9517 & 8.0718 \\
\hline & NCD & 1.9151 & 0.0646 & 0.8605 & 1.9145 & 0.0649 & 0.0427 \\
\hline \multirow{4}{*}{20} & PSNR & 25.521 & 22.4556 & 25.106 & 23.1649 & 22.7787 & 26.2477 \\
\hline & MSE & 182.3796 & 369.4194 & 200.669 & 313.757 & 342.9358 & 154.2791 \\
\hline & MAE & 10.2505 & 13.7579 & 10.3147 & 13.4039 & 13.2932 & 9.6248 \\
\hline & NCD & 1.9145 & 0.066 & 0.8606 & 1.9188 & 0.0677 & 0.0514 \\
\hline \multirow{4}{*}{25} & PSNR & 24.6924 & 22.3792 & 24.5869 & 20.5355 & 22.6064 & 24.8669 \\
\hline & MSE & 220.7213 & 375.9745 & 226.1458 & 574.8226 & 356.8122 & 212.0269 \\
\hline & MAE & 11.2366 & 13.9374 & 10.8574 & 19.1364 & 13.7181 & 11.1727 \\
\hline & NCD & 1.914 & 0.0675 & 0.8606 & 1.9486 & 0.0708 & 0.0588 \\
\hline \multirow{4}{*}{30} & PSNR & 24.0995 & 22.2744 & 24.1278 & 19.4299 & 22.4058 & 24.3301 \\
\hline & MSE & 253.0044 & 385.1557 & 251.3623 & 741.4695 & 373.6783 & 239.9205 \\
\hline & MAE & 11.9986 & 14.186 & 11.3701 & 21.7147 & 14.1787 & 11.9287 \\
\hline & NCD & 1.9125 & 0.0696 & 0.8607 & 1.9547 & 0.0742 & 0.0661 \\
\hline
\end{tabular}


The results of perceptual quality are given in Table 4 and 5. From the results it is seen that the proposed technique gives best perceptual quality results.

Table 4. Comparison of Perceptual Quality of De-noised Image (LENA)

\begin{tabular}{|l|l|l|l|l|l|l|}
\hline $\begin{array}{l}\text { Original } \\
\text { image }\end{array}$ & $\begin{array}{l}\text { Gaussian } \\
\text { noise } \sigma=5\end{array}$ & $\begin{array}{l}\text { Gaussian } \\
\text { noise } \sigma=10\end{array}$ & $\begin{array}{l}\text { Gaussian } \\
\text { noise } \sigma=15\end{array}$ & $\begin{array}{l}\text { Gaussian } \\
\text { noise } \sigma=20\end{array}$ & $\begin{array}{l}\text { Gaussian } \\
\text { noise } \sigma=25\end{array}$ & $\begin{array}{l}\text { Gaussian } \\
\text { noise } \sigma=30\end{array}$ \\
\hline & & & & & &
\end{tabular}

Table 5. Comparison of Perceptual Quality of De-noised Image (GOLDHILL)

\begin{tabular}{|c|c|c|c|c|c|c|}
\hline $\begin{array}{l}\text { Original } \\
\text { image }\end{array}$ & $\begin{array}{l}\text { Gaussian } \\
\text { noise } \sigma=5\end{array}$ & $\begin{array}{l}\text { Gaussian } \\
\text { noise } \sigma=10\end{array}$ & $\begin{array}{l}\text { Gaussian } \\
\text { noise } \sigma=15\end{array}$ & $\begin{array}{l}\text { Gaussian } \\
\text { noise } \sigma=20\end{array}$ & $\begin{array}{l}\text { Gaussian } \\
\text { noise } \sigma=25\end{array}$ & $\begin{array}{l}\text { Gaussian } \\
\text { noise } \sigma=30\end{array}$ \\
\hline 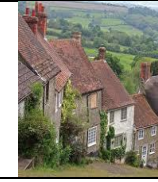 & & & & & & $x^{-}+y^{2}$ \\
\hline $\begin{array}{l}\text { Filtered b } \\
\text { BF }\end{array}$ & & & & & & \\
\hline
\end{tabular}




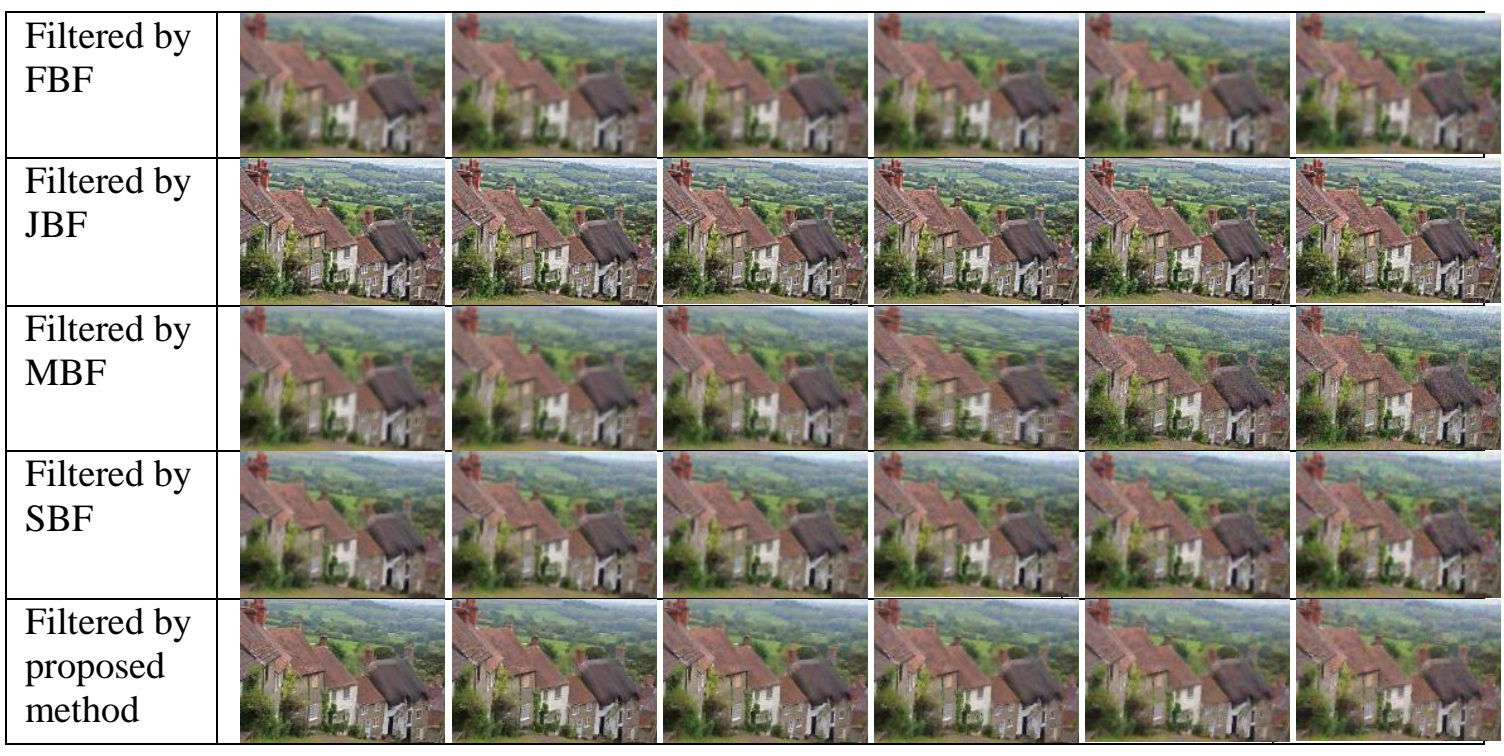

\section{Conclusion}

The following important inferences can be drawn from the above results as follows:

$>$ The proposed technique gives best results in terms of PSNR, MSE, MAE and NCD values than all other filtering techniques implemented to remove Gaussian noise.

$>$ Proposed technique provide best perceptual quality by edge sharpening and reducing the color blurriness.

$>$ The use of CIE-Lab color space smoothens the image and preserves the edges in a way that is tuned to human performance .

$>$ The proposed filtering technique works very well for dense noise and does not introduces blurriness as in case of other filtering techniques.

\section{References}

[1] C. Tomasi and R. Manduchi, "Bilateral filter for gray and color images", in Proceeding of IEEE International Conference on Computer Vision, (1998) January 4-7; Bomaby, India.

[2] K. E. Barner, Y. Nie, and W. An, "Fuzzy Ordering Theory and Its Use in Filter Generalization," EURASIP Journal of Applied Signal Processing, (2001).

[3] M. Elad, "On the Origin of the Bilateral Filter and Ways to Improve It", IEEE transactions on image processing, vol. 11, (2002).

[4] G. Petschnigg, R. Szeliski, M. Agrawala, M. Cohen, H. Hoppe, and K. Toyama, "Digital photography with flash and no-flash image pairs", In ACM Trans. Graph., vol. 23, (2004).

[5] S. Morillas, V. Gregori, and A. Sapena,Editors "Fuzzy bilateral filtering for color images," in Proceeding of International Conference on Image Analysis and Recognition, (2006).

[6] S. Schulte, V. De Witte, and E. E. Kerre,Editors “A fuzzy noise reduction method for colour images," IEEE Transactions on Image Processing, vol. 16, May (2007).

[7] S. Morillas, S. Schulte, T. Mélange, E. E. Kerre, and V. Gregori,Editors "A soft-switching approach to improve visual quality of colour image smoothing filters," in Proceeding of Advanced Concepts for Intelligent Vision Systems, (2007) August 28-31, Delft, The Netherlands.

[8] B. Zhang and J. P. Allebach, "Adaptive Bilateral Filter for Sharpness Enhancement and Noise Removal", IEEE Transactions on Image Processing, vol. 17 (2008).

[9] S. Morillas, V. Gregori, A. Herv'as, "Fuzzy peer groups for reducing mixed Gaussian impulse noise from color images", IEEE Transactions on Image Processing, vol. 18 (2009).

[10] C. H. Lin, J. S. Tsai and C. T. Chiu, "Switching bilateral filter with texture/noise detector for universal noise removal”, IEEE Transactions on Image Processing, vol. 19, (2010). 
International Journal of Signal Processing, Image Processing and Pattern Recognition

Vol.8, No.3 (2015)

[11] X. Zeng and L. Yang,Editors "Mixed impulse and Gaussian noise removal using detail-preserving regularization", Optical Engineering vol. 49 (2010), September.

[12] K. Malik and B. Smolka, "Improved Bilateral Filtering Scheme For Noise Removal in Color Images", In Proceedings of the International Conference on Informatics and Applications (2012).

[13] A. K. Tripathi and S. Mukhopadhyay, "Single image fog removal using bilateral filter," In Proceedings of IEEE International Conference on Signal Processing, Computing and Control (2012).

[14] K. Zhang, G. Lafruit, R. Lauwereins and L. Van Gool, "Constant Time Joint Bilateral Filtering Using Joint Histograms", In IEEE Transactions on Image Processing, vol. 21, (2012).

[15] R. Gonzalez and R. E. Woods, Digital Image Processing, Addison-Wesley (2002). 